\title{
Chemical Composition and Inhibitory Effects of Essential Oils on Germination and Seedling Growth of Barnyard Grass, Echinochloa crusgalli L.
}

\author{
Samir A. M. Abdelgaleil, Mona M. G. Saad, Basma Y. Hassan ${ }^{1}$
}

\begin{abstract}
Hydrodistilled essential oils from twenty plant species grown in Egypt were analyzed by gas chromatographymass spectrometry (GC-MS). The isolated oils were enriched with monoterpene hydrocarbons (i.e., limonene, sabinene, $\beta$-pinene, $\gamma$-terpinene, $\alpha$-phellandrene, $\beta$ phellandrene, $\delta$-3-carene, cis-Ocimene) and oxygenated monoterpenes (i.e., terpinen-4-ol, $\beta$-thujone, 4-terpineol, linalool, $\alpha$-citral, $\beta$-citronellol, 1,8-cineole, camphor, pulegone, a-terpinolene) except the oil of Schinus terebinthifolius which was enriched with sesquiterpenes, and the oil of Vitex agnus-castus which contained similar amounts of monoterpenes and sesquiterpenes. The inhibitory effects of the essential oils on seed germination and seedling growth of barnyard grass, Echinochloa crusgalli were examined. The oil of Myrtus communis was the most potent seed germination inhibitor at the tested concentrations $\left(625,1250,2500\right.$, and $\left.5000 \mathrm{mg} \mathrm{L}^{-1}\right)$, followed by the oils of Pelargonium graveolens, $V$. agnuscastus, Cupressus macrocarpa and $S$. terebinthifolius, while the oils of Citrus lemon, Artemisia judaica and Cupressus sempervirens were the less effective. Furthermore, the oils of $M$. communis, A. monosperma, $V$. agnus-castus and $P$. graveolens showed the highest inhibition of root and shoot growth. However, the inhibition of root growth by all essential oils was greater than that of shoot growth. These results suggest that the essential oils may serve as natural herbicidal products.
\end{abstract}

Key words: Essential oils, Egyptian plants, chemical composition, herbicidal activity, Echinochloa crusgalli

\section{INTRODUCTION}

The flora of Egypt includes about 2000 species of plants distributed in its different localities that vary in type of soil and prevailing climatic and other environmental conditions. In addition, many plants have been successfully introduced and acclimatized in Egypt (Khedr et al., 2002). Although chemical constituents and bioactivities of thousands of plants all over the world have been investigated against different pests and plant pathogens, many of Egyptian plants have not systemically been examined. Thus, it is very useful to study the chemical profiles and the biological activities of the Egyptian plants to explore their possible use in pest management programs.

Essential oils are a complex mixture of compounds, mainly monoterpenes, sesquiterpenes, and their oxygenated derivatives (alcohols, aldehydes, esters, ethers, ketones, phenols and oxides). Some volatile compounds present in essential oils include phenylpropenes and specific sulphur- or nitrogencontaining substances. Generally, essential oil composition is a balance of various compounds, although in many species one constituent may prevail over all others (Cowan, 1999). Plant essential oils, in general, have been recognized as an important natural resource of biopesticides (Gbolade et al., 2000). Their lipophilic nature facilitates their interference with basic metabolic, biochemical, physiological and behavioral functions of pests (Nishimura, 2001). Earlier studies have documented that essential oils and their constituents, mainly monoterpenoids, possess allelopathic and phytotoxic effects (Barney et al., 2005; Batish et al., 2006; Mutlu et al., 2010; Krifa et al., 2011).

Barnyard grass (Echinochloa crusgalli L.), an annual grass, is widely spread throughout the world. It has been reported to cause problems in at least 61 countries and in at least 36 different crops (Holm et al., 1991). It is a major weed in paddy fields as it competes with rice (Oryza sativa L.) and causes reduction in rice yield. It reduces crop yields by removing up to $80 \%$ of the soil nitrogen. Competition from 25 barnyard grass plants $/ \mathrm{m}^{2}$ can cause $50 \%$ reduction in rice yield (Chin, 2001).

The aims of the present study were to investigate the chemical compositions of essential oils isolated from twenty plants growing in Northern Egypt, namely, Artemisia judaica, A. monosperma, Astoma seselifolium, Callistemon viminals, Citrus aurantifolia, C. lemon, C. paradisi, C. sinensis, Cupressus macrocarpa, C. sempervirens, Myrtus communis, Origanum vulgare, Pelargonium graveolens, Pituranthos tortuosus, Rosmarinus officinalis, Syzygium cumini, Schinus molle, S. terebinthifolius, Thuja occidentalis and Vitex agnus-castus, and evaluate the inhibitory effects of these essential oils on germination and seedling growth of barnyard grass, E. crusgalli, with a view to explore them as bioherbicides for management of this weed.

\footnotetext{
${ }^{1}$ Department of Pesticide Chemistry and Technology, Faculty of Agriculture, El-Shatby, Alexandria University, Alexandria 21545, Egypt Received November 13, 2014, Accepted December 14, 2014
} 


\section{MATERIALIS AND METHODS}

\section{Plant materials}

Twenty plant species were collected during the flowering stage from different locations of Alexandria, Behira and Matrouh Governorates, Egypt, in August, 2010 to April, 2011. The plant names and their used parts are shown in Table 1 . The plant materials were identified by Prof. FathAllah Zaitoon of Plant Pathology Department, Faculty of Agriculture, Alexandria University. Voucher specimens have been deposited in Department of Pesticides Chemistry, Faculty of Agriculture, Alexandria University.

\section{Isolation of essential oils}

The aerial plant parts and leaves were partially dried at room temperature $\left(26 \pm 1^{\circ} \mathrm{C}\right)$ for five days and the fruit peels were used fresh. The plant materials were subjected to $3 \mathrm{~h}$ of hydrodistillation in a Clevenger-type apparatus. The resulting oils were dried over anhydrous sodium sulfate $\left(\mathrm{Na}_{2} \mathrm{SO}_{4}\right)$. The oil yields and their colors are presented in Table 1 . The oil samples were stored at $4{ }^{\circ} \mathrm{C}$ until used for GC-MS analysis and phytotoxic effects.

\section{Analysis of essential oils}

Analyses of essential oils were run on a gas chromatography (Hewlwett Packard 5890)/mass spectrometry (Hewlwett Packard 5989B) (GC-MS) apparatus. Essential oils were diluted in diethyl ether and the injection volume was $0.5 \mu \mathrm{l}$. The GC column was a $30 \mathrm{~m}(0.25 \mathrm{~mm}$ i.d., film thickness $0.25 \mu \mathrm{m}) \mathrm{HP}$ 5MS (5\% diphenyl) dimethylpolysiloxane capillary column. The GC conditions were as follows: injector temperature, $240^{\circ} \mathrm{C}$; column temperature, isothermal at $70^{\circ} \mathrm{C}$ and held for $2 \mathrm{~min}$, then programmed to $280^{\circ} \mathrm{C}$ at $6^{\circ} \mathrm{C} / \mathrm{min}$ and held at this temperature for $2 \mathrm{~min}$; ion source temperature, $200^{\circ} \mathrm{C}$; detector temperature, $300^{\circ} \mathrm{C}$. Helium was used as the carrier gas at the rate of $1 \mathrm{~mL} \mathrm{~min}^{-1}$. The effluent of the GC column was introduced directly into the ion source of the MS. Spectra were obtained in the EI mode with $70 \mathrm{eV}$ ionization energy. The sector mass analyzer was set to scan from 40 to $400 \mathrm{amu}$ for $5 \mathrm{~s}$. The oil components were identified by comparison of their retention indices and mass spectra with the NIST Mass Spectral Library.

\section{Test weed}

Seeds of a field biotype barnyard grass, Echinochloa crusgalli L. (Poaceae) were obtained from Faculty of Agriculture Farm, Alexandria, Egypt. Uniform seeds were selected for the test while undersized and damaged seeds were discarded. Germination of the seeds was tested before use and was $55 \%$.

\section{Seed germination and seedling growth tests}

Phytotoxic effects of the isolated essential oils were evaluated on barnyard grass (Echinochloa crusgalli) germination and subsequent seedling growth. The solutions of tested oils were first prepared in dimethyl sulfoxide (DMSO). Serial dilutions of these solutions were prepared with distilled water containing $0.02 \%$ of an emulsifying agent (Triton-X 100) to give the concentrations of $625,1250,2500$, and $5000 \mathrm{mg} \mathrm{L}^{-1}$. An aqueous solution of DMSO $(0.5 \% \mathrm{v} / \mathrm{v})$ and Triton-X $100(0.02 \%)$ was used as control treatment. Twenty seeds were placed in each Petri dishes $(9 \mathrm{~cm})$ lined with Whatman No. 2 filter paper. Three replicates were prepared for each treatment . Six milliliters of each test solution were added to the Petri dish. Afterward, Petri dishes were placed in the bottom of $0.1 \mathrm{~mm}$ thick polyethylene bags $(15 \times 30 \mathrm{~cm})$ that were expanded to contain air and then closed at the top with rubber bands to prevent the loss of moisture. The Petri dishes were kept on a germination cabinet at $26 \pm 2 \mathrm{C}$ with a $12-\mathrm{h}$ photoperiod. After 6 days of sowing, the percentages of seed germination and the lengths of root and shoot were determined. The growth inhibition percentages of root and shoot lengths were calculated from the following equation: $\mathrm{I}(\%)=[1-\mathrm{T} / \mathrm{C}] \times 100 ; \mathrm{T}$ is the root or shoot length of treatment $(\mathrm{cm})$ and $\mathrm{C}$ is the root or shoot length of control $(\mathrm{cm})$.

\section{Statistical analysis}

Germination percentages, root lengths and shoot lengths were subjected to one-way analysis of variance followed by Student-Newman-Keuls test (Cohort software Inc. 1985) to determine significant differences among mean values at the probability level of 0.05 .

\section{RESULTS}

\section{Yields and chemical compositions of the isolated essential oils}

The essential oil yields of the selected plant species obtained by hydrodistillation, on a partially dry and/or fresh weight basis, are shown in Table 1 . The oil yields ranged between $0.08 \%$ and $0.88 \%(\mathrm{v} / \mathrm{w})$. The plants of S. molle, A. monosperma, $C$. aurantifolia and $C$. sinensis contained high concentrations of oils, while the plants of S. cumini, P. graveolens, A. seselifolium and $C$. paradisi contained low concentrations of oils. The major components of the essential oils are presented in Table 1. It can be noticed that, some major components were found in more than one plant, such as limonene, $\alpha$-pinene, $\beta$-pinene, 1,8-cineole, $\beta$ citronellol, sabinene and $\gamma$-terpinene but others were specific to the plant species. The major constituents of the essential oils mainly belonged to four chemical groups: oxygenated monoterpenes (e.i., $\alpha$-thujone, $\beta$ thujone, chrysanthenone, terpinen-4-ol, linalool, 
pulegone, $\alpha$-citral, $\beta$-citronellol camphor and linalool oxide), monoterpene hydrocarbons (e.i., limonene, sabinene, $\quad \gamma$-terpinene, $\quad \beta$-pinene, $\delta$-3-carene, phellandrene and $\alpha$-pinene), sesquiterpene hydrocarbons (e.i., bicyclogermacrene, $\alpha$-elemene, $\beta$ elemene and trans-caryophyllene) and oxygenated sesquiterpenes (e.i., cedrol and elemol).
Effect of essential oils on $E$. crusgalli seed germination

The inhibitory effect of isolated oils on seed germination of E. crusgalli after 6 days of treatment is shown in Table 2. The results indicated that seed germination responses of E. crusgalli to essential oils differed significantly depending on the plant species and oil concentration. All of the tested oils caused significant reduction of seed germination particularly at the higher concentrations of 2500 and $5000 \mathrm{mg} \mathrm{L}^{-1}$.

Table 1. Major constituents and yields of essential oils isolated from twenty Egyptian plant species

\begin{tabular}{|c|c|c|c|c|}
\hline Plant name & Family & $\begin{array}{l}\text { Plant } \\
\text { part }\end{array}$ & $\begin{array}{l}\text { Oil yield }(\%) \\
(\mathrm{v} / \mathrm{w}) \text { (color) }\end{array}$ & Major components (\%) \\
\hline $\begin{array}{l}\text { Artemisia } \\
\text { judaica }\end{array}$ & Asteraceae & $\begin{array}{l}\text { Aerial } \\
\text { parts }\end{array}$ & $\begin{array}{l}0.2 \\
\text { (Pale yellow) }\end{array}$ & $\begin{array}{l}\beta-\text { Thujone (49.83), Chrysanthenone (10.88), } \alpha \text { - } \\
\text { Thujone (8.21), 1,8-Cineole (4.91) }\end{array}$ \\
\hline $\begin{array}{l}\text { Artemisia } \\
\text { monosperma }\end{array}$ & Asteraceae & Leaves & $\begin{array}{l}0.8 \\
\text { (Pale yellow) }\end{array}$ & $\begin{array}{l}\text { Capillene (36.86), capillin (14.68), } \gamma \text {-Terpinene } \\
(12.46), \beta \text {-Pinene }(7.85)\end{array}$ \\
\hline $\begin{array}{l}\text { Astoma } \\
\text { seselifolium }\end{array}$ & Apiaceae & Leaves & $\begin{array}{l}0.1 \\
\text { (Pale yellow) }\end{array}$ & $\begin{array}{l}\text { Sabinene (23.02), } \quad \text { 4-Terpineol (17.83), } \quad \gamma \text { - } \\
\text { Terpinene (8.97), Germacrene D (8.27) }\end{array}$ \\
\hline $\begin{array}{l}\text { Citrus } \\
\text { aurantifolia }\end{array}$ & Rutaceae & $\begin{array}{l}\text { Fruit } \\
\text { peels }\end{array}$ & $\begin{array}{l}0.75 \\
\text { (Colorless) } \\
\end{array}$ & $\begin{array}{l}\text { Limonene (40.19), } \beta \text {-Pinene (19.65), } \alpha \text {-Citral } \\
(8.14), \gamma \text {-Terpinene }(6.34)\end{array}$ \\
\hline Citrus limon & Rutaceae & $\begin{array}{l}\text { Fruit } \\
\text { peels }\end{array}$ & $\begin{array}{l}0.2 \\
\text { (Colorless) }\end{array}$ & $\begin{array}{l}\text { Limonene }(56.30), \beta \text {-Pinene (8.81), } \gamma \text {-Terpinene } \\
(6.42), \alpha \text {-Citral }(4.96)\end{array}$ \\
\hline $\begin{array}{l}\text { Cupressus } \\
\text { macrocarpa }\end{array}$ & Cupressaceae & Leaves & $\begin{array}{l}0.45 \\
\text { (Colorless) }\end{array}$ & $\begin{array}{l}\text { Terpinen-4-ol (20.29), Sabinene (18.67), } \beta \text { - } \\
\text { Citronellol (13.01), } \gamma \text {-Terpinene (7.59) }\end{array}$ \\
\hline Citrus paradisi & Rutaceae & $\begin{array}{l}\text { Fruit } \\
\text { peels }\end{array}$ & $\begin{array}{l}0.12 \\
\text { (Colorless) }\end{array}$ & $\begin{array}{l}\text { Limonene (74.29), Linalool (4.61), Linalool } \\
\text { oxide (4.18), } \beta \text {-Citral }(2.66)\end{array}$ \\
\hline $\begin{array}{l}\text { Cupressus } \\
\text { sempervirens }\end{array}$ & Cupressaceae & Leaves & $\begin{array}{l}0.14 \\
\text { (Colorless) }\end{array}$ & $\begin{array}{l}\alpha \text {-Pinene }(37.88), \delta \text {-Carene }(20.05), \alpha \text {-Terpinolene } \\
(6.91), \beta \text {-Myrcene }(5.47)\end{array}$ \\
\hline Citrus sinensis & Rutaceae & $\begin{array}{l}\text { Fruit } \\
\text { peels }\end{array}$ & $\begin{array}{l}0.7 \\
\text { (Colorless) }\end{array}$ & $\begin{array}{l}\text { Limonene (89.23), Linalool (2.98), } \beta \text {-Myrcene } \\
(1.77) \text {, Octanal (1.28) }\end{array}$ \\
\hline $\begin{array}{l}\text { Callistemon } \\
\text { viminals }\end{array}$ & Myrtaceae & Leaves & $\begin{array}{l}0.5 \\
\text { (Colorless) }\end{array}$ & $\begin{array}{l}\text { 1,8-Cineole (71.77), } \alpha \text {-Pinene (11.47), Terpinen- } \\
\text { 4-ol (3.18), Octadecanoic acid (3.08) }\end{array}$ \\
\hline $\begin{array}{l}\text { Myrtus } \\
\text { communis }\end{array}$ & Myrtaceae & Leaves & $\begin{array}{l}0.2 \\
\text { (Pale yellow) }\end{array}$ & $\begin{array}{l}\alpha \text {-Pinene }(26.16), 1,8 \text {-Cineole }(16.45), \text { Linalool } \\
(11.23), \beta \text {-Fenchyl alcohol }(8.34)\end{array}$ \\
\hline $\begin{array}{l}\text { Origanum } \\
\text { vulgare }\end{array}$ & Lamiaceae & $\begin{array}{l}\text { Aerial } \\
\text { parts }\end{array}$ & $\begin{array}{l}0.5 \\
(\text { Pale yellow })\end{array}$ & $\begin{array}{l}\text { Pulegone (77.45), Menthone (4.86), cis- } \\
\text { Isopulegone (2.22), Piperitenone (2.13) }\end{array}$ \\
\hline $\begin{array}{l}\text { Pelargonium } \\
\text { graveolens }\end{array}$ & Geraniaceae & Leaves & $\begin{array}{l}0.09 \\
\text { (Pale yellow) }\end{array}$ & $\begin{array}{lcc}\text {-Citronellol } \quad(35.92), & \text { Geraniol } & (11.66), \\
\text { Citronellylformate(11.40), Linalool }(9.63) & \end{array}$ \\
\hline $\begin{array}{l}\text { Pituranthos } \\
\text { tortuosus }\end{array}$ & Apiaceae & $\begin{array}{l}\text { Aerial } \\
\text { parts }\end{array}$ & $\begin{array}{l}0.22 \\
\text { (Pale yellow) }\end{array}$ & 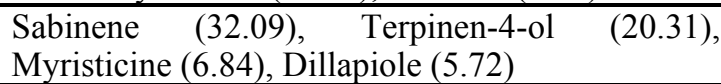 \\
\hline $\begin{array}{l}\text { Rosmarinus } \\
\text { officinalis }\end{array}$ & Lamiaceae & Leaves & $\begin{array}{l}0.33 \\
\text { (Colorless) } \\
\end{array}$ & $\begin{array}{l}\text { 1,8-Cineole (19.60), Camphor (17.01), } \alpha \text {-Pinene } \\
\text { (15.12), Verbenone (9.55) }\end{array}$ \\
\hline $\begin{array}{l}\text { Syzygium } \\
\text { cumini }\end{array}$ & Myrtaceae & Leaves & $\begin{array}{l}0.08 \\
\text { (Pale yellow) }\end{array}$ & $\begin{array}{l}\alpha \text {-Pinene }(17.26), \alpha \text {-Terpineol (13.88), } \beta \text {-Pinene } \\
(11.28) \text {, cis-Ocimene }(11.27)\end{array}$ \\
\hline Schinus molle & Anacardiaceae & Leaves & $\begin{array}{l}0.88 \\
\text { (Colorless) }\end{array}$ & $\begin{array}{l}\alpha \text {-Phellandrene (29.87), } \beta \text {-Phellandrene }(21.08) \text {, } \\
\text { Elemol (13.00), } \tau \text {-Muurolol }(5.35)\end{array}$ \\
\hline $\begin{array}{l}\text { Schinus } \\
\text { terebinthifolius }\end{array}$ & Anacardiaceae & Leaves & $\begin{array}{l}0.25 \\
\text { (Colorless) }\end{array}$ & $\begin{array}{l}\text { Sabinene (14.93), } \gamma \text {-Elemene (13.18), } \beta \text {-Elemene } \\
(6.63), \alpha \text {-Candiol }(6.61)\end{array}$ \\
\hline $\begin{array}{l}\text { Thuja } \\
\text { occidentalis }\end{array}$ & Cupressaceae & Leaves & $\begin{array}{l}0.25 \\
\text { (Pale yellow) }\end{array}$ & $\begin{array}{l}\alpha \text {-Pinene (35.49), } \delta \text {-3-Carene (25.42), } \alpha \text {-Cedrol } \\
(9.05), \alpha \text {-Terpinolene }(6.76)\end{array}$ \\
\hline $\begin{array}{ll}\text { Vitex } & \text { agnus- } \\
\text { castus } & \\
\end{array}$ & Lamiaceae & Leaves & $\begin{array}{l}0.16 \\
\text { (Yellow) }\end{array}$ & $\begin{array}{l}\text { trans-Caryophyllene (15.19), 1,8-Cineole (13.04), } \\
\text { trans- } \beta \text {-Farnesene }(8.35), 4 \text {-Terpineol }(7.45)\end{array}$ \\
\hline
\end{tabular}


Table 2. Effect of essential oils on Echinochloa crusgalli seed germination $6 \mathrm{~d}$ after sowing ${ }^{\mathrm{a}}$

\begin{tabular}{|c|c|c|c|c|c|}
\hline \multirow{3}{*}{$\begin{array}{l}\text { Conc } \\
\operatorname{mg~L}^{-1}\end{array}$} & \multicolumn{5}{|c|}{ Germination $\% \pm \mathrm{SE}$} \\
\hline & \multicolumn{5}{|c|}{ Plant oil } \\
\hline & A. Judaica & A. monosperma & A. seselifolium & C. aurantifolia & C. limon \\
\hline 0 & $55.0 \pm 2.89 \mathrm{a}^{\mathrm{b}}$ & $55.0 \pm 2.89 a$ & $55.0 \pm 2.89 a$ & $55.0 \pm 2.89 \mathrm{a}$ & $55.0 \pm 2.89 \mathrm{a}$ \\
\hline 625 & $55.0 \pm 2.89 \mathrm{a}^{\mathrm{b}}$ & $45.0 \pm 2.89 \mathrm{ab}$ & $45.0 \pm 2.89 \mathrm{~b}$ & $50.0 \pm 2.89 \mathrm{~b}$ & $50.0 \pm 2.89 \mathrm{ab}$ \\
\hline 1250 & $48.3 \pm 1.66 \mathrm{ab}$ & $43.3 \pm 1.66 \mathrm{ab}$ & $41.7 \pm 3.33 b$ & $48.3 \pm 3.31 \mathrm{~b}$ & $45.0 \pm 0.0 \mathrm{ab}$ \\
\hline 2500 & $43.3 \pm 1.66 b$ & $33.3 \pm 3.31 \mathrm{~b}$ & $38.3 \pm 3.33 b$ & $48.3 \pm 3.31 b$ & $45.0 \pm 0.0 \mathrm{ab}$ \\
\hline 5000 & $43.3 \pm 1.66 b$ & $33.3 \pm 4.41 b$ & $36.7 \pm 1.66 b$ & $31.7 \pm 1.66 b$ & $43.3 \pm 5.77 b$ \\
\hline \multirow{3}{*}{$\begin{array}{l}\text { Conc } \\
\mathrm{mg} \mathrm{L}^{-1}\end{array}$} & \multicolumn{5}{|c|}{ Germination $\% \pm \mathrm{SE}$} \\
\hline & \multicolumn{5}{|c|}{ Plant oil } \\
\hline & C. macrocarpa & C. paradisi & C. sempervirens & C. sinensis & C. viminals \\
\hline 0 & $55.0 \pm 2.89 \mathrm{a}$ & $55.0 \pm 2.89 a$ & $55.0 \pm 2.89 \mathrm{a}$ & $55.0 \pm 2.89 a$ & $55.0 \pm 2.89 \mathrm{a}$ \\
\hline 625 & $38.3 \pm 4.41 b$ & $38.3 \pm 1.66 \mathrm{~b}$ & $43.3 \pm 3.33 b$ & $40.0 \pm 2.89 b$ & $40.0 \pm 5.78 b$ \\
\hline 1250 & $38.3 \pm 1.66 b$ & $38.3 \pm 1.66 b$ & $41.7 \pm 1.66 b$ & $40.0 \pm 5.0 \mathrm{~b}$ & $40.0 \pm 2.89 \mathrm{~b}$ \\
\hline 2500 & $38.3 \pm 3.33 b$ & $35.0 \pm 5.01 \mathrm{~b}$ & $40.0 \pm 2.89 \mathrm{~b}$ & $36.7 \pm 3.33 b$ & $38.3 \pm 3.31 b$ \\
\hline 5000 & $26.7 \pm 4.41 b$ & $35.0 \pm 2.89 b$ & $40.0 \pm 0.0 \mathrm{~b}$ & $36.7 \pm 3.33 b$ & $38.3 \pm 6.01 \mathrm{~b}$ \\
\hline \multirow{3}{*}{$\begin{array}{l}\text { Conc } \\
\mathrm{mg} \mathrm{L}^{-1}\end{array}$} & \multicolumn{5}{|c|}{ Germination $\% \pm \mathrm{SE}$} \\
\hline & \multicolumn{5}{|c|}{ Plant oil } \\
\hline & M. communis & O. vulgare & P. graveolens & P. tortuosus & R. officinals \\
\hline 0 & $55.0 \pm 2.89 \mathrm{a}$ & $55.0 \pm 2.89 a$ & $55.0 \pm 2.89 \mathrm{a}$ & $55.0 \pm 2.89 a$ & $55.0 \pm 2.89 \mathrm{a}$ \\
\hline 625 & $33.3 \pm 1.66 \mathrm{~b}$ & $53.3 \pm 4.41 \mathrm{a}$ & $55.0 \pm 2.89 \mathrm{a}$ & $38.3 \pm 1.66 \mathrm{~b}$ & $40.0 \pm 0.0 \mathrm{~b}$ \\
\hline 1250 & $30.0 \pm 2.89 \mathrm{~b}$ & $31.7 \pm 1.66 \mathrm{~b}$ & $33.3 \pm 1.66 \mathrm{~b}$ & $36.7 \pm 4.41 \mathrm{~b}$ & $38.3 \pm 3.31 \mathrm{~b}$ \\
\hline 2500 & $25.0 \pm 2.89 b$ & $30.0 \pm 2.89 b$ & $28.3 \pm 3.31 b$ & $35.0 \pm 0.0 \mathrm{~b}$ & $36.7 \pm 3.31 b$ \\
\hline 5000 & $11.7 \pm 1.66 \mathrm{c}$ & $30.0 \pm 2.89 \mathrm{~b}$ & $16.7 \pm 3.31 \mathrm{c}$ & $35.0 \pm 2.89 \mathrm{~b}$ & $31.7 \pm 3.31 b$ \\
\hline \multirow{3}{*}{$\begin{array}{l}\text { Conc } \\
\mathrm{mg} \mathrm{L}^{-1}\end{array}$} & \multicolumn{5}{|c|}{ Germination $\% \pm \mathrm{SE}$} \\
\hline & \multicolumn{5}{|c|}{ Plant oil } \\
\hline & S. cumini & S. molle & S. terebinthifolius & T. occidentalis & V. agnus-castus \\
\hline 0 & $55.0 \pm 2.89 \mathrm{a}$ & $55.0 \pm 2.89 \mathrm{a}$ & $55.0 \pm 2.89 \mathrm{a}$ & $55.0 \pm 2.89 \mathrm{a}$ & $55.0 \pm 2.89 \mathrm{a}$ \\
\hline 625 & $41.7 \pm 4.41 \mathrm{~b}$ & $43.3 \pm 1.66 \mathrm{~b}$ & $38.3 \pm 4.41 b$ & $43.33 \pm 3.33 b$ & $33.3 \pm 1.66 \mathrm{~b}$ \\
\hline 1250 & $35.0 \pm 2.89 \mathrm{~b}$ & $43.3 \pm 1.66 b$ & $38.3 \pm 1.66 b$ & $43.3 \pm 1.66 \mathrm{~b}$ & $31.7 \pm 1.66 \mathrm{~b}$ \\
\hline 2500 & $31.7 \pm 1.66 b$ & $33.3 \pm 4.41 b$ & $36.7 \pm 3.33 b$ & $38.3 \pm 3.33 b$ & $31.7 \pm 4.41 b$ \\
\hline 5000 & $31.7 \pm 1.66 \mathrm{~b}$ & $31.7 \pm 1.66 \mathrm{~b}$ & $28.3 \pm 3.33 b$ & $38.3 \pm 3.33 b$ & $31.7 \pm 1.66 \mathrm{~b}$ \\
\hline
\end{tabular}

${ }^{a}$ Data are expressed as means \pm SE from experiments with three replicates of 20 seeds each.

${ }^{\mathrm{b}}$ Means within a column sharing the same letter are not significantly different at the 0.05 probability level.

The oil of $M$. communis revealed the highest reduction of seed germination at the tested concentrations. In addition, the oils of $P$. graveolens, $V$. agnus-castus, $C$. macrocarpa and $S$. terebinthifolius showed remarkable inhibition of seed germination. In contrast, the oils of $C$. lemon, A. judaica and C. sempervirens displayed the lowest inhibition of seed germination at the tested concentrations.

\section{Effect of essential oils on $\boldsymbol{E}$. crusgalli seedling growth}

The mean of root lengths and the percents of root growth inhibition of E. crusgalli treated with different concentrations of isolated oils are presented in Table 3. The results revealed that the tested oils caused reduction of root growth based on the concentration and the plant species. In the most cases, the reduction of root growth was in concentration-dependent manner. At the concentration of $625 \mathrm{mg} \mathrm{L}^{-1}$, the oils of $R$. officinalis, $C$. lemon and $A$. monosperma caused the highest reduction in root growth with inhibition percents of $54.5 \%, 45.5 \%$ and $40.9 \%$, respectively. In contrary, the oils of $O$. vulgare and $V$. agnus-castus stimulated the root growth compared with control, while the oils of $C$. macrocarpa, S. cumini and S. molle showed no root inhibition at this concentration. At the concentration of $1250 \mathrm{mg} \mathrm{L}^{-1}$, all of the tested oils inhibited root growth except the oil of $S$. molle. 
Table 3. Effect of essential oils on Echinochloa crusgalli root growth $6 \mathrm{~d}$ after sowing ${ }^{\mathrm{a}}$

\begin{tabular}{|c|c|c|c|c|c|c|c|c|}
\hline \multirow{2}{*}{$\begin{array}{l}\text { Conc } \\
\operatorname{mg~L}^{-1}\end{array}$} & \multicolumn{2}{|l|}{ A. Judaica } & \multicolumn{2}{|c|}{ A. monosperma } & \multicolumn{2}{|c|}{ A. seselifolium } & \multicolumn{2}{|c|}{ C. aurantifolia } \\
\hline & $\begin{array}{l}\text { Root length } \\
\text { (cm) }\end{array}$ & $I(\%)^{b}$ & $\begin{array}{l}\text { Root length } \\
\text { (cm) }\end{array}$ & I (\%) & $\begin{array}{l}\text { Root length } \\
\text { (cm) }\end{array}$ & I (\%) & $\begin{array}{l}\text { Root length } \\
\text { (cm) }\end{array}$ & I (\%) \\
\hline 0 & $2.2 \pm 0.11 \mathrm{a}^{\mathrm{c}}$ & 0.0 & $2.2 \pm 0.11 \mathrm{a}$ & 0.0 & $2.2 \pm 0.11 \mathrm{a}$ & 0.0 & $2.2 \pm 0.11 \mathrm{a}$ & 0.0 \\
\hline 625 & $2.1 \pm 0.29 \mathrm{a}$ & 4.5 & $1.3 \pm 0.15 b$ & 40.9 & $1.9 \pm 0.19 \mathrm{a}$ & 13.6 & $1.8 \pm 0.28 \mathrm{ab}$ & 18.2 \\
\hline 1250 & $1.5 \pm 0.12 b$ & 31.8 & $1.2 \pm 0.15 b$ & 45.5 & $1.9 \pm 0.24 \mathrm{a}$ & 13.6 & $1.5 \pm 0.07 \mathrm{~b}$ & 31.8 \\
\hline 2500 & $1.2 \pm 0.15 \mathrm{c}$ & 45.5 & $1.1 \pm 0.21 \mathrm{~b}$ & 50.0 & $0.9 \pm 0.15 b$ & 59.1 & $1.4 \pm 0.03 \mathrm{~b}$ & 36.4 \\
\hline 5000 & $0.5 \pm 0.07 \mathrm{~d}$ & 77.3 & $0.1 \pm 0.03 \mathrm{c}$ & 95.5 & $0.7 \pm 0.23 b$ & 68.2 & $0.3 \pm 0.07 \mathrm{c}$ & 86.4 \\
\hline \multirow{2}{*}{$\begin{array}{l}\text { Conc } \\
\mathrm{mg} \mathrm{L}^{-1}\end{array}$} & \multicolumn{2}{|l|}{ C. limon } & \multicolumn{2}{|c|}{ C. macrocarpa } & \multicolumn{2}{|l|}{ C. paradisi } & \multicolumn{2}{|c|}{ C. sempervirens } \\
\hline & $\begin{array}{l}\text { Root length } \\
(\mathrm{cm})\end{array}$ & I (\%) & $\begin{array}{l}\text { Root length } \\
(\mathrm{cm})\end{array}$ & I ( $\%)$ & $\begin{array}{l}\text { Root length } \\
(\mathrm{cm})\end{array}$ & I (\%) & $\begin{array}{l}\text { Root length } \\
(\mathrm{cm})\end{array}$ & I (\%) \\
\hline 0 & $2.2 \pm 0.11 \mathrm{a}$ & 0.0 & $2.2 \pm 0.11 \mathrm{a}$ & 0.0 & $2.2 \pm 0.11 \mathrm{a}$ & 0.0 & $2.2 \pm 0.11 \mathrm{a}$ & 0.0 \\
\hline 625 & $1.2 \pm 0.22 \mathrm{~b}$ & 45.5 & $2.2 \pm 0.20 \mathrm{a}$ & 0.0 & $2.0 \pm 0.03 \mathrm{a}$ & 9.1 & $2.1 \pm 0.17 \mathrm{a}$ & 4.5 \\
\hline 1250 & $1.2 \pm 0.25 \mathrm{~b}$ & 45.5 & $1.5 \pm 0.09 b$ & 31.8 & $1.4 \pm 0.15 b$ & 36.4 & $1.8 \pm 0.21 \mathrm{a}$ & 18.2 \\
\hline 2500 & $0.5 \pm 0.07 b$ & 77.3 & $1.4 \pm 0.12 b$ & 36.4 & $1.3 \pm 0.12 \mathrm{~b}$ & 40.9 & $0.9 \pm 0.22 b$ & 59.1 \\
\hline 5000 & $0.5 \pm 0.09 \mathrm{~b}$ & 77.3 & $0.6 \pm 0.12 \mathrm{c}$ & 72.7 & $0.9 \pm 0.09 \mathrm{c}$ & 59.1 & $0.6 \pm 0.19 \mathrm{~b}$ & 72.7 \\
\hline \multirow{2}{*}{$\begin{array}{l}\text { Conc } \\
\mathrm{mg} \mathrm{L}^{-1}\end{array}$} & \multicolumn{2}{|l|}{ C. sinensis } & \multicolumn{2}{|l|}{ C. viminals } & \multicolumn{2}{|l|}{ M. communis } & \multicolumn{2}{|l|}{ O. vulgare } \\
\hline & $\begin{array}{l}\text { Root length } \\
(\mathrm{cm})\end{array}$ & I (\%) & $\begin{array}{l}\text { Root length } \\
(\mathrm{cm})\end{array}$ & I (\%) & $\begin{array}{l}\text { Root length } \\
(\mathrm{cm})\end{array}$ & I (\%) & $\begin{array}{l}\text { Root length } \\
(\mathrm{cm})\end{array}$ & I (\%) \\
\hline 0 & $2.2 \pm 0.11 \mathrm{a}$ & 0.0 & $2.2 \pm 0.11 \mathrm{a}$ & 0.0 & $2.2 \pm 0.11 \mathrm{a}$ & 0.0 & $2.2 \pm 0.11 \mathrm{a}$ & 0.0 \\
\hline 625 & $1.9 \pm 0.12 \mathrm{ab}$ & 13.6 & $1.6 \pm 0.20 \mathrm{~b}$ & 27.3 & $1.8 \pm 0.12 \mathrm{ab}$ & 18.2 & $2.3 \pm 0.26 \mathrm{a}$ & -4.5 \\
\hline 1250 & $1.9 \pm 0.18 \mathrm{ab}$ & 13.6 & $1.4 \pm 0.19 b$ & 36.4 & $1.6 \pm 0.20 \mathrm{~b}$ & 27.3 & $1.5 \pm 0.26 \mathrm{~b}$ & 31.8 \\
\hline 2500 & $1.4 \pm 0.19 b c$ & 36.4 & $1.3 \pm 0.17 \mathrm{~b}$ & 40.9 & $0.5 \pm 0.07 \mathrm{c}$ & 77.3 & $1.1 \pm 0.19 \mathrm{~b}$ & 50.0 \\
\hline 5000 & $1.3 \pm 0.12 \mathrm{c}$ & 40.9 & $0.6 \pm 0.07 \mathrm{c}$ & 72.7 & $0.1 \pm 0.02 \mathrm{~d}$ & 95.5 & $0.7 \pm 0.35 \mathrm{~b}$ & 68.2 \\
\hline \multirow{2}{*}{$\begin{array}{l}\text { Conc } \\
\mathrm{mg} \mathrm{L}^{-1}\end{array}$} & \multicolumn{2}{|l|}{ P. graveolens } & \multicolumn{2}{|l|}{ P. tortuosus } & \multicolumn{2}{|l|}{ R. officinals } & \multicolumn{2}{|l|}{ S. cumini } \\
\hline & $\begin{array}{l}\text { Root length } \\
\text { (cm) }\end{array}$ & I (\%) & $\begin{array}{l}\text { Root length } \\
(\mathrm{cm})\end{array}$ & I (\%) & $\begin{array}{l}\text { Root length } \\
\text { (cm) }\end{array}$ & I (\%) & $\begin{array}{l}\text { Root length } \\
\text { (cm) }\end{array}$ & I (\%) \\
\hline 0 & $2.2 \pm 0.11 \mathrm{a}$ & 0.0 & $2.2 \pm 0.11 \mathrm{a}$ & 0.0 & $2.2 \pm 0.11 \mathrm{a}$ & 0.0 & $2.2 \pm 0.11 \mathrm{a}$ & 0.0 \\
\hline 625 & $2.1 \pm 0.22 \mathrm{a}$ & 4.5 & $1.9 \pm 0.23 \mathrm{a}$ & 13.6 & $1.0 \pm 0.09 \mathrm{~b}$ & 54.5 & $2.2 \pm 0.20 \mathrm{a}$ & 0.0 \\
\hline 1250 & $1.2 \pm 0.42 \mathrm{~b}$ & 45.5 & $1.8 \pm 0.15 \mathrm{a}$ & 18.2 & $0.9 \pm 0.10 \mathrm{bc}$ & 59.1 & $1.5 \pm 0.15 b$ & 31.8 \\
\hline 2500 & $0.6 \pm 0.10 \mathrm{c}$ & 72.7 & $1.7 \pm 0.12 \mathrm{a}$ & 22.7 & $0.9 \pm 0.15 \mathrm{bc}$ & 59.1 & $1.3 \pm 0.22 \mathrm{~b}$ & 40.9 \\
\hline 5000 & $0.2 \pm 0.06 \mathrm{c}$ & 90.9 & $0.9 \pm 0.13 b$ & 59.1 & $0.5 \pm 0.12 \mathrm{c}$ & 77.3 & $0.4 \pm 0.10 \mathrm{c}$ & 81.8 \\
\hline \multirow{2}{*}{$\begin{array}{l}\text { Conc } \\
\mathrm{mg} \mathrm{L}^{-1}\end{array}$} & \multicolumn{2}{|l|}{ S. molle } & \multicolumn{2}{|c|}{ S. terebinthifolius } & \multicolumn{2}{|c|}{ T. occidentalis } & \multicolumn{2}{|c|}{ V. agnus-castus } \\
\hline & $\begin{array}{l}\text { Root length } \\
\text { (cm) }\end{array}$ & I (\%) & $\begin{array}{l}\text { Root length } \\
(\mathrm{cm})\end{array}$ & I (\%) & $\begin{array}{l}\text { Root length } \\
\text { (cm) }\end{array}$ & I (\%) & $\begin{array}{l}\text { Root length } \\
\text { (cm) }\end{array}$ & I (\%) \\
\hline 0 & $2.2 \pm 0.11 \mathrm{a}$ & 0.0 & $2.2 \pm 0.11 \mathrm{a}$ & 0.0 & $2.2 \pm 0.11 \mathrm{a}$ & 0.0 & $2.2 \pm 0.11 \mathrm{a}$ & 0.0 \\
\hline 625 & $2.2 \pm 0.13 \mathrm{a}$ & 0.0 & $2.1 \pm 0.31 \mathrm{a}$ & 4.5 & $2.1 \pm 0.23 \mathrm{~b}$ & 4.5 & $2.4 \pm 0.09 \mathrm{a}$ & -9.1 \\
\hline 1250 & $2.2 \pm 0.17 \mathrm{a}$ & 0.0 & $1.9 \pm 0.06 \mathrm{a}$ & 13.6 & $2.1 \pm 0.12 b$ & 4.5 & $1.6 \pm 0.23 b$ & 27.3 \\
\hline 2500 & $0.9 \pm 0.18 \mathrm{~b}$ & 59.1 & $1.6 \pm 0.18 \mathrm{a}$ & 27.3 & $1.6 \pm 0.15 b$ & 27.3 & $1.1 \pm 0.12 \mathrm{c}$ & 50.0 \\
\hline 5000 & $0.3 \pm 0.12 \mathrm{c}$ & 86.4 & $1.0 \pm 0.15 \mathrm{~b}$ & 54.5 & $1.0 \pm 0.12 b$ & 54.5 & $0.2 \pm 0.06 \mathrm{~d}$ & 90.9 \\
\hline
\end{tabular}

${ }^{a}$ Data are expressed as means \pm SE from experiments with three replicates of 20 seeds each.

${ }^{\mathrm{b}} \mathrm{I}=$ inhibition.

${ }^{\mathrm{c}}$ Means within a column sharing the same letter are not significantly different at the 0.05 probability level.

The oil of $R$. officinalis revealed the highest root growth inhibition $(59.1 \%)$, followed by the oils of $A$. monosperma, C. lemon and $P$. graveolens. The oils displayed strong root growth inhibition at concentrations of 2500 and $5000 \mathrm{mg} \mathrm{L}^{-1}$. The inhibition percents of $E$. crusgalli root growth ranged between $22.7 \%$ and $77.7 \%$ at concentration of $2500 \mathrm{mg} \mathrm{L}^{-1}$, and ranged between $40.9 \%$ and $95.9 \%$ at concentration of $5000 \mathrm{mg} \mathrm{L}^{-1}$.

The essential oils of $M$. communis, A. monosperma and $V$. agnus-castus showed pronounced shoot growth inhibition of E. crusgalli at the tested concentrations (Table 4). In contrary, the oils of $A$. seselifolium, $C$. sempervirens and T. occidentalis displayed weak shoot growth inhibition without significant differences 
between the treatments and control. The other oils showed moderate shoot growth inhibition.

Table 4. Effect of essential oils on Echinochloa crusgalli shoot growth $6 \mathrm{~d}$ after sowing ${ }^{\mathrm{a}}$

\begin{tabular}{|c|c|c|c|c|c|c|c|c|}
\hline \multirow{2}{*}{$\begin{array}{l}\text { Conc } \\
\mathrm{mg} \mathrm{L}^{-1}\end{array}$} & \multicolumn{2}{|l|}{ A. Judaica } & \multicolumn{2}{|c|}{ A. monosperma } & \multicolumn{2}{|c|}{ A. seselifolium } & \multicolumn{2}{|l|}{ C. aurantifolia } \\
\hline & $\begin{array}{ll}\text { Shoot length } \\
\text { (cm) }\end{array}$ & $I(\%)^{b}$ & $\begin{array}{l}\text { Shoot length } \\
\text { (cm) }\end{array}$ & I (\%) & $\begin{array}{l}\text { Shoot length } \\
\text { (cm) }\end{array}$ & I (\%) & $\begin{array}{l}\begin{array}{l}\text { Shoot length } \\
\text { (cm) }\end{array} \\
\end{array}$ & I (\%) \\
\hline 0 & $4.6 \pm 0.12 \mathrm{a}^{\mathrm{c}}$ & 0.0 & $4.6 \pm 0.12 \mathrm{a}$ & 0.0 & $4.6 \pm 0.12 \mathrm{a}$ & 0.0 & $4.6 \pm 0.12 \mathrm{a}$ & 0.0 \\
\hline 625 & $3.8 \pm 0.10 \mathrm{~b}$ & 17.9 & $3.3 \pm 0.0 \mathrm{~b}$ & 28.3 & $4.6 \pm 0.10 \mathrm{a}$ & 0.0 & $3.6 \pm 0.22 b$ & 21.7 \\
\hline 1250 & $3.6 \pm 0.10 \mathrm{~b}$ & 21.7 & $3.3 \pm 0.03 \mathrm{~b}$ & 28.3 & $4.5 \pm 0.19 \mathrm{a}$ & 2.2 & $3.5 \pm 0.35 b$ & 23.9 \\
\hline 2500 & $3.4 \pm 0.18 \mathrm{~b}$ & 26.1 & $3.2 \pm 0.06 \mathrm{~b}$ & 30.4 & $4.3 \pm 0.13 \mathrm{a}$ & 6.5 & $3.4 \pm 0.24 \mathrm{~b}$ & 26.1 \\
\hline 5000 & $2.5 \pm 0.24 \mathrm{c}$ & 45.7 & $1.6 \pm 0.17 \mathrm{c}$ & 65.2 & $4.1 \pm 0.09 \mathrm{a}$ & 10.9 & $2.3 \pm 0.20 \mathrm{c}$ & 50.0 \\
\hline \multirow{2}{*}{$\begin{array}{l}\text { Conc } \\
\mathrm{mg} \mathrm{L}^{-1}\end{array}$} & \multicolumn{2}{|l|}{ C. limon } & \multicolumn{2}{|c|}{ C. macrocarpa } & \multicolumn{2}{|l|}{ C. paradisi } & \multicolumn{2}{|c|}{ C. sempervirens } \\
\hline & $\begin{array}{ll}\begin{array}{l}\text { Shoot length } \\
(\mathrm{cm})\end{array} \\
\end{array}$ & I (\%) & $\begin{array}{l}\text { Shoot length } \\
(\mathrm{cm})\end{array}$ & I (\%) & $\begin{array}{l}\text { Shoot length } \\
(\mathrm{cm})\end{array}$ & I (\%) & $\begin{array}{ll}\begin{array}{l}\text { Shoot } \\
\text { (cm) }\end{array} & \text { length } \\
\end{array}$ & I (\%) \\
\hline 0 & $4.6 \pm 0.12 \mathrm{a}$ & 0.0 & $4.6 \pm 0.12 \mathrm{a}$ & 0.0 & $4.6 \pm 0.12 \mathrm{a}$ & 0.0 & $4.6 \pm 0.12 \mathrm{a}$ & 0.0 \\
\hline 625 & $4.0 \pm 0.30 \mathrm{ab}$ & 13.0 & $4.0 \pm 0.22 b$ & 13.0 & $3.9 \pm 0.33 \mathrm{ab}$ & 15.2 & $4.3 \pm 0.10 \mathrm{a}$ & 6.5 \\
\hline 1250 & $3.5 \pm 0.26 \mathrm{bc}$ & 23.9 & $4.0 \pm 0.22 b$ & 13.0 & $3.8 \pm 0.22 \mathrm{ab}$ & 17.9 & $4.2 \pm 0.09 \mathrm{a}$ & 8.7 \\
\hline 2500 & $3.2 \pm 0.21 \mathrm{~cd}$ & 30.4 & $3.6 \pm 0.09 \mathrm{~b}$ & 21.7 & $3.6 \pm 0.15 b$ & 21.7 & $3.8 \pm 0.29 a$ & 17.9 \\
\hline 5000 & $2.7 \pm 0.06 \mathrm{c}$ & 41.3 & $2.7 \pm 0.09 \mathrm{c}$ & 41.3 & $3.3 \pm 0.20 \mathrm{~b}$ & 28.7 & $3.8 \pm 0.26 \mathrm{a}$ & 17.9 \\
\hline \multirow{2}{*}{$\begin{array}{l}\text { Conc } \\
\mathrm{mg} \mathrm{L}^{-1}\end{array}$} & \multicolumn{2}{|l|}{ C. sinensis } & \multicolumn{2}{|c|}{ C. viminals } & \multicolumn{2}{|c|}{ M. communis } & \multicolumn{2}{|l|}{ O. vulgare } \\
\hline & $\begin{array}{ll}\begin{array}{l}\text { Shoot length } \\
\text { (cm) }\end{array} \\
\end{array}$ & I (\%) & $\begin{array}{l}\text { Shoot length } \\
(\mathrm{cm})\end{array}$ & I (\%) & $\begin{array}{l}\text { Shoot length } \\
(\mathrm{cm})\end{array}$ & I (\%) & $\begin{array}{ll}\begin{array}{l}\text { Shoot length } \\
(\mathrm{cm})\end{array} \\
\end{array}$ & I (\%) \\
\hline 0 & $4.6 \pm 0.12 \mathrm{a}$ & 0.0 & $4.6 \pm 0.12 \mathrm{a}$ & 0.0 & $4.6 \pm 0.12 \mathrm{a}$ & 0.0 & $4.6 \pm 0.12 \mathrm{a}$ & 0.0 \\
\hline 625 & $3.8 \pm 0.1 \mathrm{~b}$ & 17.9 & $3.9 \pm 0.27 b$ & 15.2 & $3.7 \pm 0.01 \mathrm{~b}$ & 19.6 & $4.1 \pm 0.07 \mathrm{~b}$ & 10.9 \\
\hline 1250 & $3.7 \pm 0.06 \mathrm{~b}$ & 19.6 & $3.4 \pm 0.22 b$ & 26.1 & $2.8 \pm 0.17 \mathrm{c}$ & 39.1 & $3.9 \pm 0.19 b c$ & 15.2 \\
\hline 2500 & $3.4 \pm 0.03 \mathrm{bc}$ & 26.1 & $3.3 \pm 0.25 b$ & 28.3 & $1.9 \pm 0.15 \mathrm{~d}$ & 58.7 & $3.4 \pm 0.25 c$ & 26.1 \\
\hline 5000 & $3.2 \pm 0.15 \mathrm{c}$ & 30.4 & $3.3 \pm 0.27 \mathrm{~b}$ & 28.3 & $0.7 \pm 0.10 \mathrm{c}$ & 84.8 & $3.4 \pm 0.10 \mathrm{c}$ & 26.1 \\
\hline \multirow{2}{*}{$\begin{array}{l}\text { Conc } \\
\mathrm{mg} \mathrm{L}^{-1}\end{array}$} & \multicolumn{2}{|l|}{ P. graveolens } & \multicolumn{2}{|l|}{ P. tortuosus } & \multicolumn{2}{|l|}{ R. officinals } & \multicolumn{2}{|l|}{ S. cumini } \\
\hline & $\begin{array}{ll}\begin{array}{l}\text { Shoot length } \\
(\mathrm{cm})\end{array} \\
\end{array}$ & I ( $\%)$ & $\begin{array}{l}\text { Shoot length } \\
\text { (cm) }\end{array}$ & I ( $\%)$ & $\begin{array}{l}\text { Shoot length } \\
(\mathrm{cm})\end{array}$ & I (\%) & $\begin{array}{l}\text { Shoot length } \\
\text { (cm) }\end{array}$ & I ( $\%)$ \\
\hline 0 & $4.6 \pm 0.12 \mathrm{a}$ & 0.0 & $4.6 \pm 0.12 \mathrm{a}$ & 0.0 & $4.6 \pm 0.12 \mathrm{a}$ & 0.0 & $4.6 \pm 0.12 \mathrm{a}$ & 0.0 \\
\hline 625 & $4.3 \pm 0.12 \mathrm{a}$ & 6.5 & $3.9 \pm 0.27 \mathrm{~b}$ & 15.2 & $3.7 \pm 0.19 b$ & 19.6 & $3.6 \pm 0.27 \mathrm{~b}$ & 21.7 \\
\hline 1250 & $3.6 \pm 0.19 \mathrm{~b}$ & 21.7 & $3.8 \pm 0.19 \mathrm{~b}$ & 17.9 & $2.8 \pm 0.07 \mathrm{c}$ & 39.1 & $3.3 \pm 0.12 b$ & 28.3 \\
\hline 2500 & $3.5 \pm 0.10 \mathrm{~b}$ & 23.9 & $3.6 \pm 0.27 \mathrm{~b}$ & 21.7 & $2.8 \pm 0.07 \mathrm{c}$ & 39.1 & $3.1 \pm 0.09 \mathrm{~b}$ & 32.6 \\
\hline 5000 & $2.2 \pm 0.23 \mathrm{c}$ & 52.2 & $2.9 \pm 0.28 \mathrm{~b}$ & 37.0 & $2.6 \pm .23 \mathrm{c}$ & 43.5 & $2.5 \pm 0.12 \mathrm{c}$ & 45.7 \\
\hline \multirow{2}{*}{$\begin{array}{l}\text { Conc } \\
\mathrm{mg} \mathrm{L}^{-1}\end{array}$} & \multicolumn{2}{|l|}{ S. molle } & \multicolumn{2}{|c|}{ S. terebinthifolius } & \multicolumn{2}{|c|}{ T. occidentalis } & \multicolumn{2}{|c|}{ V.agnus-castus } \\
\hline & $\begin{array}{l}\text { Shoot length } \\
\text { (cm) }\end{array}$ & I (\%) & $\begin{array}{l}\text { Shoot length } \\
\text { (cm) }\end{array}$ & I (\%) & $\begin{array}{l}\text { Shoot length } \\
(\mathrm{cm})\end{array}$ & I (\%) & $\begin{array}{l}\text { Shoot length } \\
(\mathrm{cm})\end{array}$ & I (\%) \\
\hline 0 & $4.6 \pm 0.12 \mathrm{a}$ & 0.0 & $4.6 \pm 0.12 \mathrm{a}$ & 0.0 & $4.6 \pm 0.12 \mathrm{a}$ & 0.0 & $4.6 \pm 0.12 \mathrm{a}$ & 0.0 \\
\hline 625 & $3.9 \pm 0.21 \mathrm{~b}$ & 15.2 & $4.4 \pm 0.10 \mathrm{a}$ & 4.3 & $4.2 \pm 0.26 \mathrm{a}$ & 8.7 & $2.9 \pm 0.25 b$ & 37.0 \\
\hline 1250 & $3.8 \pm 0.17 \mathrm{~b}$ & 17.9 & $3.7 \pm 0.15 b$ & 19.6 & $4.1 \pm 0.09 a$ & 10.9 & $2.9 \pm 0.25 b$ & 37.0 \\
\hline 2500 & $3.4 \pm 0.07 \mathrm{bc}$ & 26.1 & $3.7 \pm 0.15 b$ & 19.6 & $3.7 \pm 0.28 \mathrm{a}$ & 19.6 & $2.7 \pm 0.06 \mathrm{~b}$ & 41.3 \\
\hline 5000 & $3.0 \pm 0.20 \mathrm{c}$ & 34.8 & $3.3 \pm 0.27 b$ & 28.3 & $3.7 \pm 0.22 \mathrm{a}$ & 19.6 & $2.1 \pm 0.06 \mathrm{~b}$ & 54.3 \\
\hline
\end{tabular}

${ }^{\mathrm{a}}$ Data are expressed as means \pm SE from experiments with three replicates of 20 seeds each.

${ }^{\mathrm{b}} \mathrm{I}=$ inhibition.

${ }^{\mathrm{c}}$ Means within a column sharing the same letter are not significantly different at the 0.05 probability level.

\section{DISCUSSION}

The chemical compositions of the isolated essential oils from $C$. aurantifolia, C. paradise, C. limon, $C$. sinensis, $C$. viminals, $C$. sempervirens, $S$. molle, $C$. macrocarpa, $P$. graveolens, $R$. officinals and $M$. communis are in accordance with those previously reported in literature (Lota et al., 2001; Srivastava et al.,
2003; Tuberoso et al., 2006; Viuda-Martos et al., 2009; Bendaoud et al., 2010). The oil of $A$. seselifolium was analysed for the first time in this study. On the other hand, the major constituents of the essential oils isolated from $A$. monosperma, $O$. vulgare, $T$. occidentalis and $A$. judaica were completely differed with those previously reported on the chemistry of these oils (Şahin et al., 
2004; Mohamed and Abdelgaleil, 2008; Tsiri et al., 2009; Khan et al., 2012). Some of the major constituents of the essential oils of $P$. tortuosus, V. agnus-castus, $S$. terebinthifolius and $S$. cumini were similar to those previously reported for the oils isolated from plants growing in Egypt and other countries around the world (Singab et al., 2003; Gundidza et al., 2009; Stojković et al., 2011). However, the percentages of the major constituents are differed. The chemical composition of essential oils of the same plants may vary widely depending on geographical location, season, environmental conditions and nutritional status of the plants (Perry et al., 1999).

All of the tested oils showed seed germination inhibition against E. crusgalli. In general, our results agree with those reported in the literature on inhibitory activity exerted by some of tested essential oils on seed germination of other plant and weed species. For example, the oils of $S$. molle, A. judaica, $R$. officinals, $O$. vulgare and $P$. graveolens were described to possess seed germination inhibition of wheat, Triticum aestivum L. (Dudai et al., 1999; Zahed et al., 2010). The oil of $R$. officinals showed inhibitory effect on seed germination of Cynodon dactylon L., Festuca arundinacea L. and Lolium peranne L. (Saharkhiz et al., 2009). In addition, the oil of $O$. vulgare was reported to inhibit seed germination of Raphanus sativus L., Lactuca sativa L. and Lepidium sativum (Arminante et al., 2006). Similarly, Amri et al. (2013) demonstrated that the oil of $C$. sempervirens reduced the seed germination of Sinapis arvensis L., Phalaris paradoxa L. and Raphanus raphanistrum L. Also, the inhibitory effect of essential oils from other plants on seed germination was previously reported (Barbosa et al., 2007; Paudel and Gupta, 2008; Kordali et al., 2009).

The results of the inhibitory effects of the isolated oils on the seedling growth showed that the oils caused higher inhibitory effect on root growth than shoot growth. These results are consistent with those reported elsewhere for plant metabolites and extracts (Abdelgaleil et al., 2009; Saad et al., 2012). This finding might be expected, because it is likely that roots are the first to absorb the allelochemical compounds from the environment (Turk and Tawaha, 2002).

To the best of our knowledge, this is the first report on the herbicidal activity of the twenty isolated oils against E. crusgalli. However, some of essential oils isolated from the tested plants were documented to possess allelopathic and herbicidal activities. Zahed et al. (2010) stated that the oil of $S$. molle had inhibitory effect on the seedling growth of wheat. Similarly, the oil of $R$. officinalis reduced the seedling growth of of Cynodon dactylon L., Festuca arundinacea L. and
Lolium peranne L. (Saharkhiz et al., 2009). Moreover, the oil of $O$. vulgare inhibited the radicle growth of Raphanus sativus L., Lactuca sativa L. and Lepidium sativum L. (Almeida et al., 2010). The inhibitory effect of the essential oils of $C$. sempervirens and $C$. sinensis on the seedling growth of common crop weeds was described (Amri et al., 2013; Ribeiro and Lima, 2012). In addition, the herbicidal and phytotoxic activities of other essential oils were described (Scrivanti et al., 2003; Singh et al., 2009; Mutlu et al., 2010).

The results of the present study indicated that there is a correlation between the chemical compositions of the essential oils and their bioactivities. Thus, the essential oils with high content of oxygenated monoterpenes showed stronger phytotoxic effects than the essential oils with low content of oxygenated monoterpenes and high content of monoterpene hydrocarbons. This finding is in congruent with earlier studies on the bioactivity of essential oils and monoterpenes in which the potent inhibition of seed germination and seedling growth was linked to the present of high percentage of oxygenated monoterpenes (Scrivanti et al., 2003; Almeida et al., 2010).

It is clear that the allelopathic activity of the essential oils resulted from the combined effects of several allelochemicals, mainly monoterpenoids and sesquiterpenoids, including addition, synergism and antagonism (Kordali et al., 2009). The modes of action of the allelopathic activity of the essential oils remain unclear. However, several studies were described the possible mechanisms of action of essential oils and monoterpenes. These mechanisms include, DNA synthesis inhibition, disruption of membranes surrounding mitochondria and nuclei, uncoupling oxidative phosphorylation, inhibition of electron transfer, inhibition of mitochondrial ATP production, inhibition of mitochondrial reparation, reduction of chlorophyll contents; inhibition of cell proliferation and disruption of the activity of metabolic enzymes involved in glycolysis (Nishida et al., 2005; Singh et al., 2006; Macias et al., 2007; Kaur et al., 2010).

Based on the results of the present study, it can be concluded that the essential oils of $M$. communis, $A$. monosperma, $V$. agnus-castus and $P$. graveolens had remarkable herbicidal activity against $E$. crusgalli. Such results provide evidences for the possible utilization of these oils as bioherbicides in future.

\section{REFERENCES}

Abdelgaleil, S. A. M.; N. Abdel-Razeek and S. A. Soliman (2009). Herbicidal activity of three sesquiterpene lactones on wild oat (Avena fatua) and their possible mode of action. Weed Sci. 57:6-9. 
Almeida, L. F. D.; F. Frei; E. Mancini; L. De Martino and V. De Feo (2010). Phytotoxic activities of Mediterranean essential oils. Molecules 15: 4309-4323.

Amri, I.; M. Hanana; S. Gargouri; B. Jamoussi and L. Hamrouni (2013). Comparative study of two coniferous species (Pinus pinaster Aiton and Cupressus sempervirens L. var. dupreziana [A. Camus] Silba) essential oils: chemical composition and biological activity. Chil. J. Agric. Res. 73: 259-266.

Arminante. F.; E. De Falco; V. De Feo; L. De Martino; E. Mancini and E. Quaranta (2006) Allelopathic activity of essential oils from Mediterranean Labiatae. Acta Horticulturae (ISHS) 723: 347-356.

Barbosa, L. C. A.; A. J. Demuner; A. D. Clemente; V. F. Paula and F. M. D. Ismail (2007) Seasonal variation in the composition of volatile oils from Schinus terebinthifolius Raddi. Química Nova 30: 1959-1965.

Barney, J. N.; A. G. Hay and L. A. Weston (2005) Isolation and characterization of allelopathic volatiles from mugwort (Artemisia vulgaris). J. Chem. Ecol. 31: $247-$ 265.

Batish, D. R.; H. P. Singh; N. Setia; S. Kaur and R. K. Kohli (2006) Chemical composition and phytotoxicity of volatile essential oil from intact and fallen leaves of Eucalyptus citriodora. Zeitschrift für Naturforschung 61c: 465-471.

Bendaoud, H.; M. Romdhane; J. P. Souchard; S. Cazaux; and J. Bouajila (2010) Chemical composition and anticancer and antioxidant activities of Schinus molle L. and Schinus terebinthifolius Raddi aerries essential oils. J. Food Sci. 75: 466-472.

Chin, D. V. (2001) Biological management of barnyard grass, red sprangletop and weedy rice. Weed Biol. Manag. 1: $37-41$.

Cohort Software Inc. (1985) Costat User's Manual. Version 3. Tucson, AZ: Cohort.

Cowan, M.M. 1999. Plant products as antimicrobial agents. Clin. Microbiol. Rev. 12: 564-582.

Dudai, N.; A. Poljakoff-Mayber; M. Mayer; E. Putievsky and H. R. Lerner (1999) Essential oils as allelochemicals and their potential use as bioherbicides. J. Chem. Ecol. 25: 1079-1089.

Gbolade, A. A.; A. O. Oyedele; M. B. Sosan; F. B. Adewayin and O. L. Soyela (2000) Mosquito repellent activities of essential oils from two Nigerian Ocimum species. J. Trop. Med. Plants 1: 146-148.

Gundidza, M.; N. Gweru; M. L. Magwa; V. Mmbengwa and A. Samie (2009) The chemical composition and biological activities of essential oil from the fresh leaves of Schinus terebinthifolius from Zimbabwe. Afr. J. Biotechnol. 8: 7164-7169.

Holm, G. L.; D. L. Plucknett; J. V. Pancho and J. P. Herber (1991) The world's worst weeds-Distribution and ecology. Krieger Publishing Company, Malabar, FL, USA pp 32, 341, p 609.
Kaur, S.; H. P. Singh; S. Mittal; D. R. Batish and R. K. Kohli (2010) Phytotoxic effects of volatile oil from Artemisia scoparia against weeds and its possible use as a bioherbicide. Ind. Crops Prod. 32: 54-61.

Khan, M.; A. A. Mousa; K. V. Syamasundar and H. Z. Alkhathlan (2012) Determination of chemical constituents of leaf and stem essential oils of Artemisia monosperma from central Saudi Arabia. Nat. Prod. Commun. 7: 1079-1082.

Khedr A.; M. W. Cadotte; A. El-Keblawy and J. Lovettdoust (2002) Phylogenetic diversity and ecological features in the Egyptian flora. Biodiver. Conserv. 11: 1809-1824.

Kordali, S.; A. Cakir, T. A. Akcin; E. Mete; A. Akcin; T. Aydin and H. Kilic (2009) Antifungal and herbicidal properties of essential oils and $n$-hexane extracts of Achillea gypsicola Hub-Mor. and Achillea biebersteinii Afan. (Asteraceae). Ind. Crops Prod. 29: 562-570.

Krifa, M.; T. Gharad and R. Haouala (2011) Biological activities of essential oil, aqueous and organic extracts of Pituranthos tortuosus (Coss.) Maire. Scientia Horticulturae 128: 61-67.

Lota, M. L.; D. De Rocca Serra; C. Jacquemon; F. Tomi and J. Casanova (2001) Chemical variability of peel and leaf essential oils of sour orange. Biochem. Syst. Ecol. 16: $89-96$.

Macías, F. A.; J. M. G. Molinillo; R. M. Varela and J. G. G. Galindo (2010) Allelopathy - a natural alternative for weed control. Pest Manag. Sci. 63: 327-348.

Mohamed, M. I. E. and S. A. M. Abdelgaleil (2008) Chemical composition and insecticidal potential of the essential oils from Egyptian plants against Sitophilus oryzae (L.) (Coleoptera: Curculionidae) and Tribolium castaneum (Herbst) (Coleoptera: Tenebrionidae). J. Appl. Entomol. Zool. 43: 599-607.

Mutlu, S.; O. Atici and N. Esim (2010) Bioherbicidal effects of the essential oils of Nepeta meyeri Benth. on weed spp. Allelopathy J. 26: 291-300.

Nishida, N.; S. Tamotsu; N. Nagata; C. Saito and A. Sakai (2005). Allelopathic effects of volatile monoterpenoids produced by Salvia leucophylla: inhibition of cell proliferation and DNA synthesis in the root apical meristem of Brassica campestris seedlings. J. Chem. Ecol. 31: 1187-1203.

Nishimura, H. (2001) Aroma constituents in plants and their repellent activities against mosquitoes. Aroma Res. 2: 257-267.

Paudel, V. R. and V. N. P. Gupta (2008) Effect of some essential oils on seed germination and seedling length of Parthenium hysterophorous L. Ecoprint 15: 69-73.

Perry, N. B.; R. E. Anderson; N. J. Brennan; M. H. Douglas; A. J. Heaney; J. A. McGrimpsey and B. M. Smallfield (1999) Essential oil from Dalmation sage (Salvia officinalis L.), variations among individuals, plant parts, seasons and sites. J. Agric. Food Chem. 47: 2048-2054. 
Ribeiro, J. P. N. and M. I. S. Lima (2012). Allelopathic effects of orange (Citrus sinensis L.) peel essential oil. Acta Bot. Bras. 26: 256-259.

Saad M. M. G.; S. A. M. Abdelgaleil and T. Suganuma (2012) Herbicidal potential of pseudoguaninolide sesquiterpenes on wild oat, Avena fatua L. Biochem. Syst. Ecol. 44: 333-337.

Saharkhiz, M. J.; F. Ashiri; M. R. Salehi; J. Ghaemaghami and S. Mohammadi (2009) Allelopathic potentialof essential oils from Carum copticum L., Cuminum cyminum L., Rosmarinus officinalis L. and Zataria multiflora Boiss. Med. Aroma. Plant Sci. Biotechnol. 3: $32-35$.

Şahin, F.; M. Güllüce; D. Daferera; A. Sökmen; M. Sökmen; M. Polissiou; G. Agar and H. Özer (2004) Biological activities of the essential oils and methanol extract of Origanum vulgare ssp. vulgare in the Eastern Anatolia region of Turkey. Food Cont. 15: 549-557.

Scrivanti, L. R.; M. Zunino and J. A. Zygadlo (2003) Tagetes minuta and Schinus areira essential oils as allelopathic agents. Biochem. Syst. and Ecol. 31: 563-572.

Singab, A. B. (2003) Essential oils and lipids content of Pituranthos species growing in Egypt. Bull Fac. Pharm. Cairo Univ. 41: 213-217.

Singh, H. P.; S. Kaur; S. Mittal; D. R. Batish and R. K. Kohli (2009) Essential oil of Artemisia scoparia inhibit plant growth by generating reactive oxygen species and causing oxidative damage. J. Chem. Ecol. 35: 154-162.

Singh, S. P.; D. R. Batish; S. Kaur; K. Arora and R. K. Kohli (2006) $\alpha$-Pinene inhibits growth and induces oxidative stress in roots. Ann. Bot. 98: 1261-1269.
Srivastava, S. K.; A. Ahmad; K. V. Syamsunder; K. K. Aggarwal and S. P. S. Khanuja (2003) Essential oil composition of Callistemon viminalis leaves from India. Flavour Frag. J. 18: 361-363.

Stojković, D.; M. Soković; J. Glamočlija; A. Džamić; A. Ćirić; M. Ristić and M. Grubišić (2011) Chemical composition and antimicrobial activity of Vitex agnus-castus L. fruits and leaves essential oils. Food Chem. 128: 1017-1022.

Tsiri, D.; K. Graikou; L. Pobłocka-Olech; M. KrauzeBaranowska; C. Spyropoulos and I. Chinou (2009) Chemosystematic value of the essential oil composition of Thuja species cultivated in Poland- antimicrobial activity. Molecules 14: 4707-4715.

Tuberoso, C. I. G.; A. Barra; A. Angioni; E. Sarritzu and F. M. Pirisi (2006) Chemical composition of volatiles in Sardinian Myrtle (Myrtus communis L.) alcoholic extracts and essential oils. J. Agric. Food Chem. 54: $1420-1426$.

Turk, M. A. and A. M. Tawaha (2002) Inhibitory effects of aqueous extracts of black mustard on germination and growth of lentil. Pak. J. Agron. 1: 28-30.

Viuda-Martos, M.; Y. Ruiz-Navajas; J. Fernández-López and J. A. Pérez-Álvarez, (2009). Chemical composition of mandarin (C. reticulata L.), grapefruit (C. paradisi L.), lemon (C. limon L.) and orange (C. sinensis L.) essential oils. J. Essent. Oil Bearing Plants 12: 236-243.

Zahed, N.; K. Hosni; N. B. Brahim; M. Kallel and H. Sebei (2010) Allelopathic effect of Schinus molle essential oils on wheat germination. Acta Physiologiae Plantarum 32: $1221-1227$. 


\section{الملغص العرب}

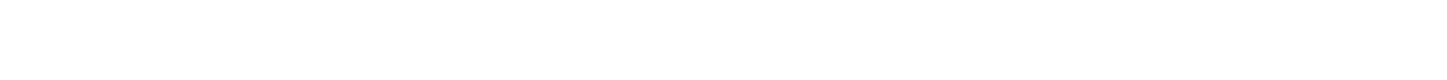

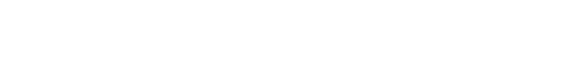

الدنية. ألطهرت النتائج أن زيت نبلت الميرسين كلن ألكثر

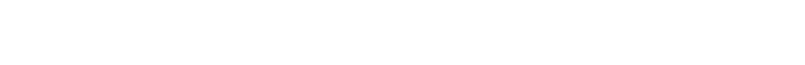

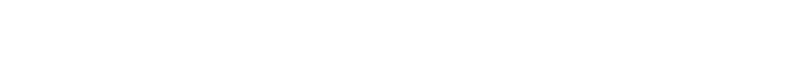

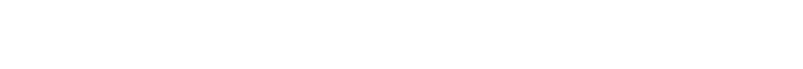

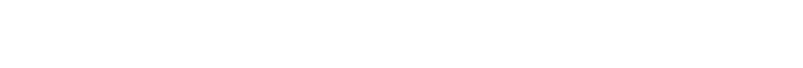

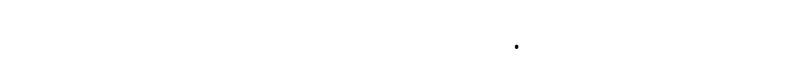
الميسسن واللل وكف مريم والعتر البلدى أعلى الزيوت الئل

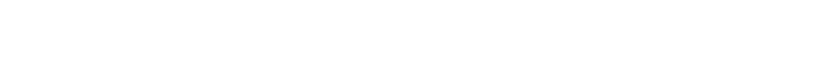

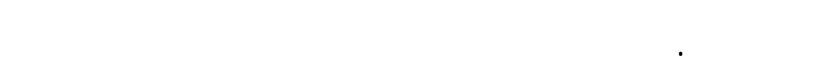

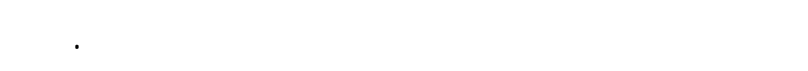

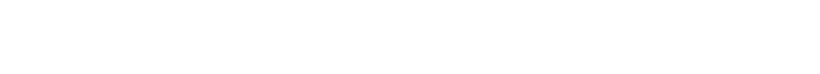

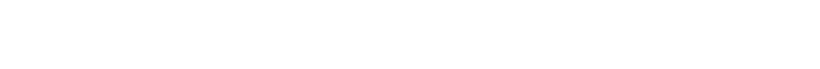
الدنيبة ويمكن أن تستخدم كمركبلت طبيعية فى مكافحة

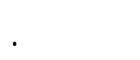

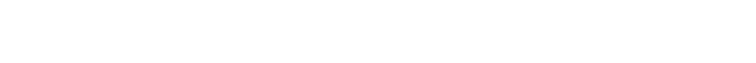
مصر والمتحصل عليها بالنطير المائى بولنطة جهاز النطاز كروماتوجرافى الغاز المتصل بمطيف الكتلة (GC-MS). أوضصت النتائج أن الزيوت المعزولة كالفت المناف غنية بمركبلت

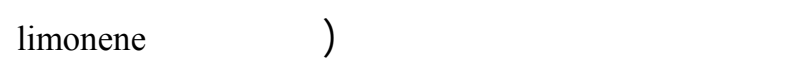
$\alpha$-phellandreneg $\gamma$-terpineneg $\beta$-pineneg sabinene 9 (cis-Ocimene $g$ 8-3-careneg $\beta$-phellandreneg والتربينت الأحاية المحتوية على الأوكسجين (مل مركبلت linaloolg 4-terpineolg $\beta$-thujoneg terpinen-4-ol camphorg 1,8-cineoleg $\beta$-citronellolg $\alpha$-citralg (a-terpinoleneg pulegoneg)، عدا زيت الفلفل عريض

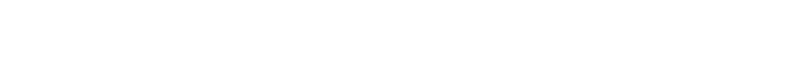

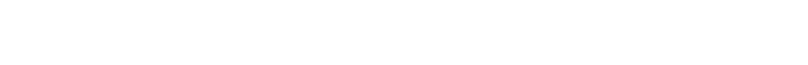

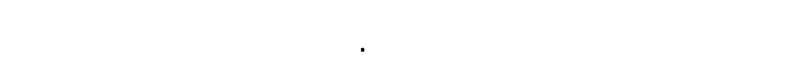
للزيوت المعزولة على إنبلت بذور ونمو بادرات هشيشة 